\title{
Effects of Aldose Reductase Inhibitors on the Progression of Nerve Fiber Damage in Diabetic Neuropathy
}

\author{
Douglas A. Greene
}

\begin{abstract}
Aldose reductase inhibitors improve nerve biochemistry, function, and structure in diabetic animals and increase nerve conduction in diabetic patients. Nevertheless, it has been difficult to demonstrate a benefit from these agents in patients with clinically overt diabetic neuropathy. Direct measurement of the nerve tissue penetration and biochemical and biological potency of these compounds is essential to fully understand and evaluate their effectiveness. Human sural nerve biopsies obtained from diabetic neuropathic patients undergoing treatment with an aldose
\end{abstract}

reductase inhibitor revealed a reduction in intermediates of the polyol pathway. Specific morphologic lesions that correlate with the degree of clinical and electrophysiologic impairment also were identified. Morphologic evaluation of sural nerve biopsies obtained after aldose reductase inhibitor treatment suggests that these biochemically effective compounds ameliorate clinically relevant structural lesions in patients with diabetic neuropathy. (Journal of Diabetes and Its Complications 6;1:35-38, 1992.)

\section{INTRODUCTION}

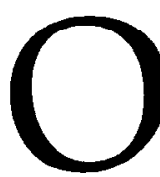
Only a decade ago, the long-term neurologic complications of diabetes mellitus were attributed to nerve degeneration or demyelination. More recently, attention has focused on a potentially reversible metabolic disorder in which the enzyme, aldose reductase, in response to hyperglycemia, promotes increased intercellular conversion of glucose to sorbitol and decreased uptake of the cyclic hexanol, myo-inositol. ${ }^{1,2}$ The depletion of myo-inositol in nerve tissue appears responsible for a variety of metabolic and electrophysiologic alterations, including a decrease in sodium-

Reprint requests to be sent to: Dr. Douglas A. Greene, Michigan Diabetes Research and Training Center, University of Michigan, 1150 West Medical Center Drive, Ann Arbor, MI 48109, USA.

Michigan Diabetes Research and Training Center, University of Michigan, Ann Arbor, Michigan, USA potassium adenosine triphosphatase (ATPase), a decrease in energy consumption of the nerve tissue, andalterations in nerve conduction. As a result, it appears that intra-axonal sodium accumulates at the node of Ranvier and causes localized swelling and the characteristic lesion, axo-glial dysjunction. The pharmacologic inhibition of aldose reductase represents an opportunity to reduce sorbitol accumulation and to arrest or reverse nerve damage that occurs as a complication of diabetes mellitus.

Aldose reductase inhibitors block the conversion of glucose to sorbitol, the depletion of myo-inositol, and the impairment of sodium-potassium ATPase; ${ }^{2}$ however, their effectiveness in ameliorating clinically overt diabetic neuropathy is uncertain. Short-term studies with aldose reductase inhibitors have not been uniformly effective. ${ }^{3-9}$ It may be that the accumulation of sorbitol contributes little to the chronic nerve fiber damage that is characteristic of diabetic neuropathy. Long-term studies with aldose reductase inhibi- 
tors that focus on outcome measures of nerve fiber structure and density are more likely to provide insights into the etiology and pathology of chronic symptomatic diabetic neuropathies. Further, detailed measurements of changes in nerve fiber parameters can provide an effective means of comparing different aldose reductase inhibitors.

\section{STRUCTURAL ABNORMALITIES ASSOCIATED WITH DIABETIC NEUROPATHY}

Three structural abnormalities, the so-called triopathy of diabetic neuropathy, are observed in patients with diabetic neuropathy: fiber loss, fiber atrophy, and specific fiber lesions. Patients with diabetic polyneuropathy demonstrate a reduced number of functional myelinated nerve fibers, and those fibers that survive are reduced in size. This decrease in axonal size is exhibited as a reduction in cross-sectional area.

The three components of the triopathy provide a series of measurable and quantifiable morphometric parameters which exhibit a relationship to the duration of diabetes in patients with diabetic neuropathy. For example, fiber loss can be quantified as a decrease in fiber density, measured as the number of fibers per square millimeter of a cross-sectional area of nerve. Fiber atrophy can be characterized several ways; these include a decrease in the mean fiber size, an increase in the myelin endpoint, or a decrease in the ratio of axonal to myelin area. Fiber atrophy in an axon can be demonstrated by changes in the relationship between the area of the axon and the number of myelin lamella. Because myelin assumes a wrinkled appearance when atrophied, increases in the percentage of wrinkled myelin is also evidence of fiber atrophy.

Specific fiber lesions can be identified by the accumulation of a series of discrete abnormalities on micrographs or as a decrease in the percentage of normal-appearing fibers. Many of these changes occur initially at the node of Ranvier, and one of the earliest lesions is the axo-glial dysjunction. This lesion is described as a loss of tight junction complexes that normally maintain the integrity of the node of Ranvier by anchoring terminal loops of myelin to the axolemma. More advance abnormalities that occur are swelling of the node and perinodal region and perinodal demyelination followed by remyelination. Frequently, increased segmental demyelination also occurs. In response to these injuries, increased neurofiber regeneration may be demonstrated with regenerating sprouts occupying the area derived from the previously degenerated axon.

It is possible to quantify the overall degree of pathology that is present by using a variety of different scoring systems. One method uses a compound parameter score (density, atrophy, and lesions) that is normalized for age and gender. ${ }^{10}$ This approach clearly distinguishes patients with diabetic peripheral polyneuropathy from a normal reference population. Further, diabetics without symptomatic peripheral neuropathy appear to be grouped with the larger normal population, rather than with the patients having diabetic polyneuropathy. This system allows one to differentiate between neuropathic and non-neuropathic diabetics on the basis of a threshold of pathology.

A similar system has been developed at our institution. It is called the index of normality and is obtained from the product of fiber density and the percentage of fibers with normal appearance. ${ }^{2}$ This method differs from that of Dyck et al. ${ }^{10}$ in that it does not correct for age and gender. Using this and other quantitative measures, we have investigated the effects of different aldose reductase inhibitors on nerve fiber morphology.

\section{THE EFFECTS OF ALDOSE REDUCTASE INHIBITORS ON NERVE FIBER MORPHOLOGY}

Three long-term, double-blind, randomized, controlled studies of aldose reductase inhibitors are available for the morphologic examination of human nerve specimens. In each study, sural nerve biopsies were obtained prior to and after at least 12 months of therapy, and morphometric techniques were employed to quantify nerve fiber changes.

Sorbinil North American Neuropathy Trial. Sural nerve biopsy specimens were obtained from 16 diabetics with symptomatic peripheral polyneuropathy prior to and 12 months after sorbinil $250 \mathrm{mg}$ per day $(n=10)$ or placebo $(n=6)$ therapy. $^{2}$ Although the mean sural nerve sorbitol concentration in sorbiniltreated patients was significantly decreased when compared with baseline measurements, no significant change was noted in placebo-treated patients. Of note, sural nerve sorbitol levels actually increased in one patient who discontinued the drug. This clearly indicates that sorbinil enters peripheral nerve tissue at the site of aldose reductase and decreases sorbitol accumulation by inhibiting polyol activity.

A number of morphometric findings were available from this study. Fiber loss was partially corrected, as indicated by a statistically significant increase in nerve fiber density in sorbinil-treated patients and no change in the placebo-treated patients. The increase in nerve fiber density was accounted for by a severalfold increase in nerve fiber regeneration. It should be noted that the failure to observe deterioration of nerve fiber density in placebo-treated patients during the 12-month study is consistent with the slow progression of diabetic polyneuropathy.

The number of specific fiber lesions, including axoglial dysjunction, were increased at baseline in both the placebo and sorbinil groups. During the study, these returned to normal in the sorbinil group but 
were unchanged in the placebo group. Similarly, axonal atrophy, as evidenced by either the axon/myelin ratio or the presence of excessive myelin wrinkling, was improved after sorbinil, indicating that aldose reductase inhibition exerted a beneficial effect on fiber atrophy. Sorbinil-treated patients had an increase in the overall percentage of nerve fibers that showed a normal appearance due to the repair of previously damaged fibers. This increase in normality was corrected for the increase in regeneration, and thus, actually represents repair of previously damaged neurons.

When this data was combined into the overall score for index of normality, an improvement in the index of normality was noted with sorbinil, as compared with no change in the placebo group. This indicates that biochemically effective aldose reductase inhibitors appear to produce consistent improvement in the structural abnormalities associated with diabetic neuropathy.

Statil North American Neuropathy Trial. In this double-blind, controlled study, sural nerve biopsy specimens were obtained from 47 neuropathic diabetics prior to and 18 months after treatment with statil $600 \mathrm{mg}$ per day $(n=21)$ or placebo $(n=26) .^{11}$ No significant change was observed in nerve sorbitol concentrations or in the index of normality in either statil- or placebo-treated patients. This suggests that statil did not achieve tissue levels in the peripheral nerve that were adequate to inhibit aldose reductase. Consistent with the lack of a biochemical effect, there was no improvement in any morphometric parameters with statil compared with placebo.

Tolrestat United States Trial. This was an openlabel study in which single sural nerve biopsies were obtained after 3.5-4 years of treatment with tolrestat. ${ }^{12}$ Patients were then entered into a 12-month double-blind, randomized continuation or withdrawal treatment period. A sural nerve biopsy was obtained at the end of the 12-month period. Eighteen neuropathic patients with long-standing diabetes (duration of approximately 25 years) who were treated for 3-5 years with tolrestat $200-400 \mathrm{mg}$ per day in the openlabel study were randomized to continue receiving tolrestat $(n=9)$ or were switched to placebo treatment $(n=9)$. These patients tended to be older and predominantly insulin-dependent diabetics; thus, it can be assumed that they had advanced peripheral neuropathy prior to entering the study.

Sorbitol and glucose concentration measurements from sural nerve biopsies obtained prior to and after 12 months of therapy showed that tolrestat inhibited sural nerve sorbitol accumulation. In placebo-treated patients, there was a glucose-mediated rise in sural nerve sorbitol levels. Peak sorbitol concentrations reached $3.5-5.5 \mathrm{nmol} / \mathrm{mg}$ protein in patients whose glucose nerve concentrations were between 250 and $350 \mathrm{nmol} / \mathrm{mg}$ protein. In contrast, sorbitol concentrations in tolrestat-treated patients reached a plateau at between 1 and $1.5 \mathrm{nmol} / \mathrm{mg}$ protein, despite sural nerve glucose concentrations that ranged from less than 75 to almost $250 \mathrm{nmol} / \mathrm{mg}$ protein. A decrease in the glucose-mediated rise in fructose also was noted with tolrestat treatment but not with placebo, which indicates a significant inhibition of the polyol pathway by tolrestat.

No statistically significant differences were revealed in a comparison of nerve morphometry in patients receiving open-label tolrestat who were randomly reassigned to double-blind treatment with tolrestat or placebo. This is expected based on the slow rate of progression of diabetic neuropathy. There was, however, a statistically significant relationship between the duration of treatment with tolrestat and the extent of nerve fiber regeneration. Furthermore, a negative correlation was observed between the duration of treatment with tolrestat and axo-glial dysjunction, which suggests that prolonged treatment with tolrestat stimulated fiber regeneration and prevented worsening or improved axo-glial dysjunction.

A more-extensive evaluation of nerve fiber morphology was conducted using tolrestat-treated patients from the United States study and comparing them with two reference populations of untreated diabetic patients entering other aldose reductase inhibitor trials. Recently, this reference population was expanded by the addition of younger patients with a shorter duration of diabetes.

Three statistical approaches were used to correct for differences in age and duration of diabetes between the groups. The data for the reference populations were adjusted with age and nondiabetic controls for each of the morphometric parameters. Data also were adjusted for age and duration of diabetes. Finally, a complex analysis of the expanded diabetic reference population was performed using a quadratic surface adjustment paradigm to look at within group relationships regarding both age and duration of diabetes.

A significant increase in fiber regeneration was observed in untreated diabetics when compared with nondiabetic controls. This increase would be expected from any patient with diabetic peripheral polyneuropathy. However, tolrestat treatment was associated with a fourfold increase in fiber regeneration compared with untreated diabetics. Axo-glial dysjunction was significantly increased in untreated diabetics with polyneuropathy, as compared with nondiabetic controls, and was essentially normal after tolrestat treatment. This indicates that tolrestat had corrected the increase in axo-glial dysjunction that was seen in the other reference populations. Similarly, segmental de- 
myelination, which was increased in the reference population compared with nondiabetic controls, was normalized in patients who were treated with tolrestat for 3.5-4 years.

Analysis of the effects of tolrestat on fiber density was more complex because fiber density is a reflection of the course of diabetes over many years, which makes adjustments for age and duration of diabetes difficult. Compared with untreated diabetics, there was a trend for improvement in fiber density in the tolrestat group. But when the larger reference population-which included many patients with less severe neuropathy-was used for comparison, there appeared to be a decrease in fiber density in the tolrestat group; thus, on the basis of these patient populations, it is not possible to make a conclusion about the effect of tolrestat on nerve fiber density.

\section{CONCLUSION}

These data support the contention that nerve fiber regeneration is a sensitive and consistent marker of tissue-specific biological activity of aldose reductase inhibitors in clinical and overt diabetic peripheral polyneuropathy. Data from long-term studies with two of the three aldose reductase inhibitors presented herein demonstrate a broad spectrum of morphometric improvements that are indicative of overall tissue repair. This data strongly argues for the role of the polyol pathway in the pathogenesis of clinically overt diabetic peripheral polyneuropathy and maintains the hope that treatment with aldose reductase inhibitors will reduce the progression or improve and partially reverse neuropathy even at late stages when the patient is clinically impaired.

\section{REFERENCES}

1. Greene DA, Lattimer SA, Sima AAF: Sorbitol, phosphoinositides, and sodium-potassium-ATPase in the pathogenesis of diabetic complications. $N$ Engl J Med 316:599-606, 1987

2. Sima AAF, Bril V, Nathaniel V, McEwen TAJ, Brown
MB, Lattimer SA, Greene DA: Regeneration and repair of myelinated fibers in sural-nerve biopsy specimens from patients with diabetic neuropathy treated with sorbinil. N Engl J Med 319:548-555, 1988.

3. Fagius J, Brattber A, Jameson S, Berne C: Limited benefit of treatment of diabetic polyneuropathy with an aldose reductase inhibitor: A 24-week controlled trial. Diabetologia 28:323-329, 1985.

4. Young RJ, Ewing DJ, Clarke BF: A controlled trial of sorbinil, an aldose reductase inhibitor, in chronic painful diabetic neuropathy. Diabetes 32:938-942, 1983.

5. Lewin IG, O'Brien IAD, Morgan $\mathrm{MH}$, Corrall RJM: Clinical and neurophysiological studies with the aldose reductase inhibitor, sorbinil, in symptomatic diabetic neuropathy. Diabetologia 26:445-448, 1984.

6. Lehtinen JM, Hyvönen SK, Uusitpa M, Pahakainen E, Halonen T, Kilpeläinen $\mathrm{H}$ : The effect of sorbinil treatment on red cell sorbitol levels and clinical and electrophysiologic parameters of diabetic neuropathy. I Neurol 223:174-177, 1986.

7. Christensen JEJ, Varnek L, Gregersen G: The effect of an aldose reductase inhibitor (Sorbinil (B) on diabetic neuropathy and neural function of the retina: A double-blind study. Acta Neurol Scand 71:164-167, 1985.

8. Fagius J, Jameson S: Effects of aldose reductase inhibitor treatment in diabetic polyneuropathy-a clinical and neurophysiological study. I Neurol Neurosurg Psychiatry 44:991-1001, 1981

9. Handelsman DJ, Turtle JR: Clinical trial of an aldose reductase inhibitor in diabetic neuropathy. Diabetes 30:459-464, 1981.

10. Dyck PJ, Karnes JL, Daube J, O'Brien P, Service FJ: Clinical and neuropathological criteria for the diagnosis and staging of diabetic polyneuropathy. Brain 108:861-880, 1985.

11. Greene DA, Sima AAF, Statil North American Painful Neuropathy Study Group: Efficacy of aldose reductase inhibitors in human sural nerve (abstract), presented at symposium, Controversies in Etiology \& Treatment of Diabetic Neuropathy. New York, June 1991.

12. Greene DA, Bochenek $W$, Harati $Y$, Sima AAF, Hohman T, Hicks D, Beg M, Gonen B, Tolrestat Study Group: Biochemical and morphometric response to tolrestat in human diabetic nerve (abstract), presented at European Association for the Study of Diabetes. Copenhagen, September 1990. 